\title{
Cor Triatriatum in an Adult
}

\section{Anish Hirachan, ${ }^{1}$ Dipanker Prajapati, ${ }^{2}$ Madhu Roka, ${ }^{2}$ Murari Dhungana, ${ }^{2}$ Deewakar Sharma ${ }^{2}$}

\author{
${ }^{1}$ National Academy of Medical Sciences, Kathmandu \\ ${ }^{2}$ Sahid Gangalal National Heart Centre, Bansbari, Kathmandu \\ Corresponding author: Anish Hirachan \\ Department of Cardiology, \\ National Academy of Medical Sciences, Bir Hospital, Mahaboudha, Kathmandu \\ Email address: hirachananish@gmail.com
}

Abstract

A 29 year old female patient presented to the cardiology OPD with history of progressive breathlessness of NYHA class II and palpitation of 1 year duration. Under evaluation, she underwent 2D transthoracic echocardiography that revealed an extra septum that subdivided the left atrium into proximal and distal chambers. The diagnosis of cor triatriatum was hence made and was referred to surgical team for corrective surgery. The communication between proximal and distal chamber was provided by large fenestration in the fibromuscular membrane.

Keywords : Cor triatriatum, fenestration , left atrium

\section{Introduction}

Cor triatriatum is a congenital anomaly that was first reported by Church in $1868 .{ }^{1}$ Cor triatriatum sinister (CTS) is a rare congenital anomaly that is caused by a fibromuscular membrane dividing the left atrium (LA) into two chambers. Communication between the pulmonary veins and anterior chamber is provided by fenestrations on the membrane. As a result of atrial flow obstruction, symptoms of congestive heart failure develop during infancy and childhood. When the membrane is incomplete or has large fenestrations, the condition is diagnosed at adulthood due to lack of symptoms. In this anomaly, the pulmonary veins drain into the upper portion of the corresponding atrium, whereas the atrial appendage and the true atrial septum are components of the inferior portion. The inferior or distal part terminates to atrioventricular valve. Cor triatriatum is generally diagnosed in pediatric population, with age of presentation depending on the size of membrane fenestration and the resulting obstruction to inflow. ${ }^{2}$

\section{Case Report}

A 29 year old female without any significant family history presented to our out patient clinic with history of progressive breathlessness of NYHA class II along with history of recurrent palpitations since one year duration. On general examination; she had $\mathrm{BP}$ of $100 / 70 \mathrm{mmHg}$, pulse rate of $70 \mathrm{bpm}$ and her cardiovascular examination revealed normal S1 with loud S2 with no obvious murmurs heard. ECG revealed sinus rhythm with normal axis and no evidence of chamber enlargement. Her 2D and colour Doppler echocardiography revealed a membrane in the left atrium dividing it into two chambers along with a fenestration noted in the membrane along which a transmembrane peak gradient of $21 \mathrm{~mm} \mathrm{Hg}$ was present along with mild tricuspid regurgitation with TRPG of $44 \mathrm{mmHg}$ (PASP $44+10=54 \mathrm{mmHg}$ ), dilated right atrium and left atrium (Fig 1, 2 and 3). She was put on oral diuretics and referred for surgical correction.

\section{Discussion:}

Cor triatriatum is a rare congenital heart disease (CHD), $0.1 \%$ of all congenital cardiac defects but a higher incidence, up to $0.4 \%$ has been reported in autopsies of patients with CHD. ${ }^{3}$ It's a surgically correctable CHD and can occur as an isolated defect (classic) or in association with other congenital cardiac anomalies (atypical). ${ }^{4}$ This condition is a result of failure of embryologic common pulmonary vein incorporation into the back of the LA. As a result, the LA is divided into two chambers by a fibromuscular membrane. The two cavities, positioned posterior superior and anterior inferior, are anatomically and functionally separated. ${ }^{5}$ Other cardiac anomalies that accompany CTS are ventricular septal defect, coarctation of aorta, tetralogy of Fallot, atrioventricular septal defect, and mitral stenosis. Depending on the severity of obstruction, CTS may be symptomatic or asymptomatic. Symptoms are as a result of LV inflow obstruction and include dyspnoea, orthopnoea, haemoptysis, and chest discomfort. . Some cases may remain asymptomatic or minimally symptomatic during lifetime. Rarely, obstructive symptoms may became manifest during adulthood. ${ }^{6}$

Our patient developed obstructive symptoms at the age of 29 and had a fenestrated membrane dividing the left atrium with the gradient of $21 \mathrm{mmHg}$ along with features of moderate pulmonary hypertension for which she was referred for an early surgical correction.

\section{Conclusion}

Cor triatriatum sinister can be observed in adulthood when the dividing membrane is incomplete. Echocardiography can readily demonstrate the membrane, while $3 \mathrm{D}$ reconstruction allows complete anatomical evaluation. Surgical removal is indicated when obstructive symptoms are present, while management for asymptomatic individuals still remains unknown. 


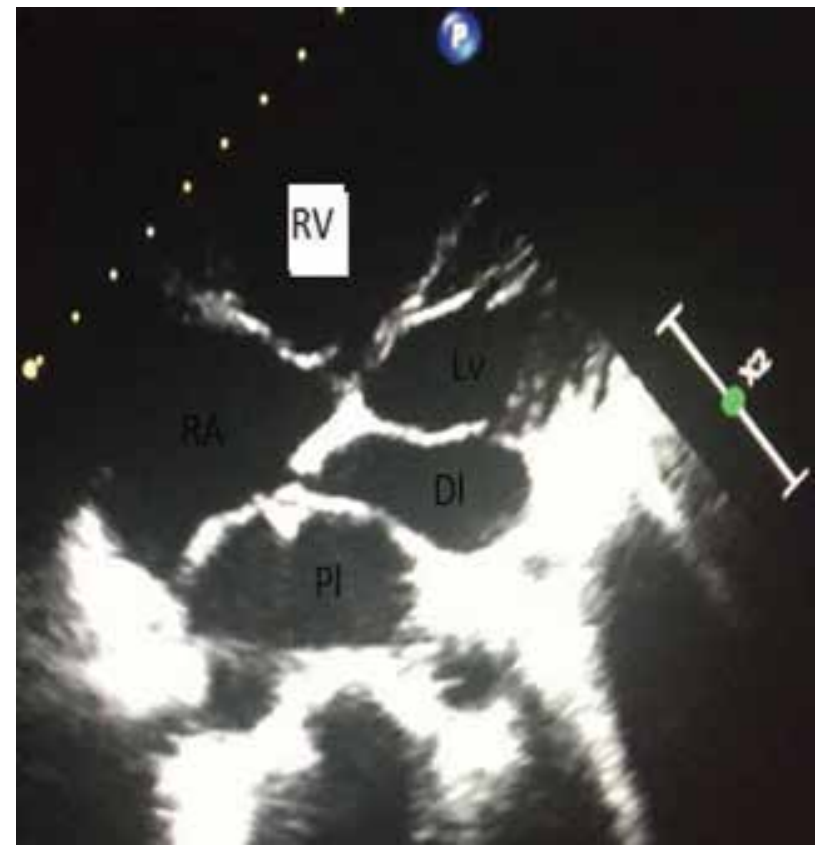

Fig 1. Apical 4- chamber view showed membrane across left atrium (across proximal ,Pl and Dl distal chamber of left atrium)

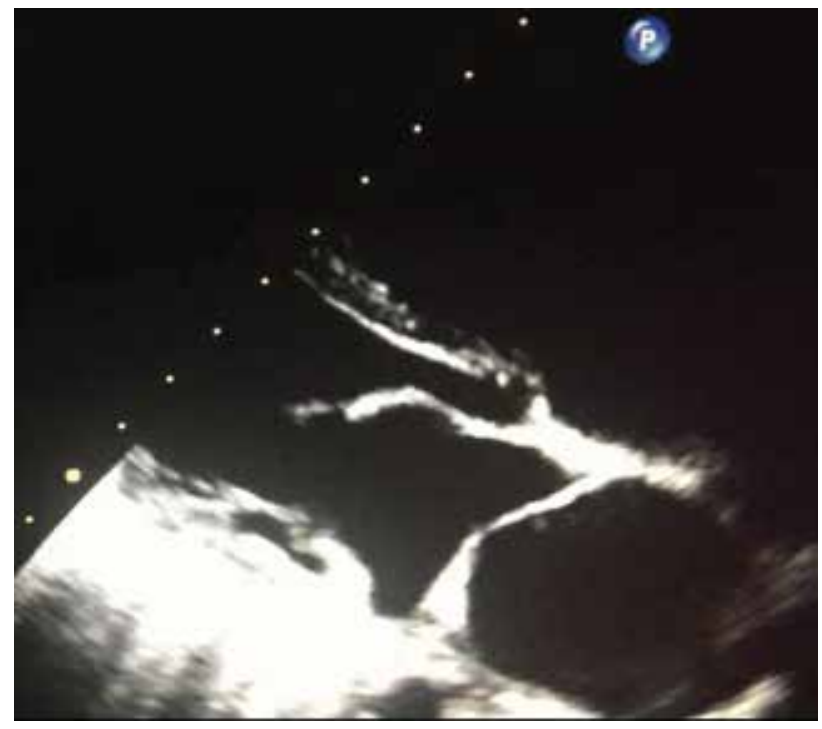

Fig 2. 2D Transthoracic echo : Parasternal Long Axis view showing cor triatriatum

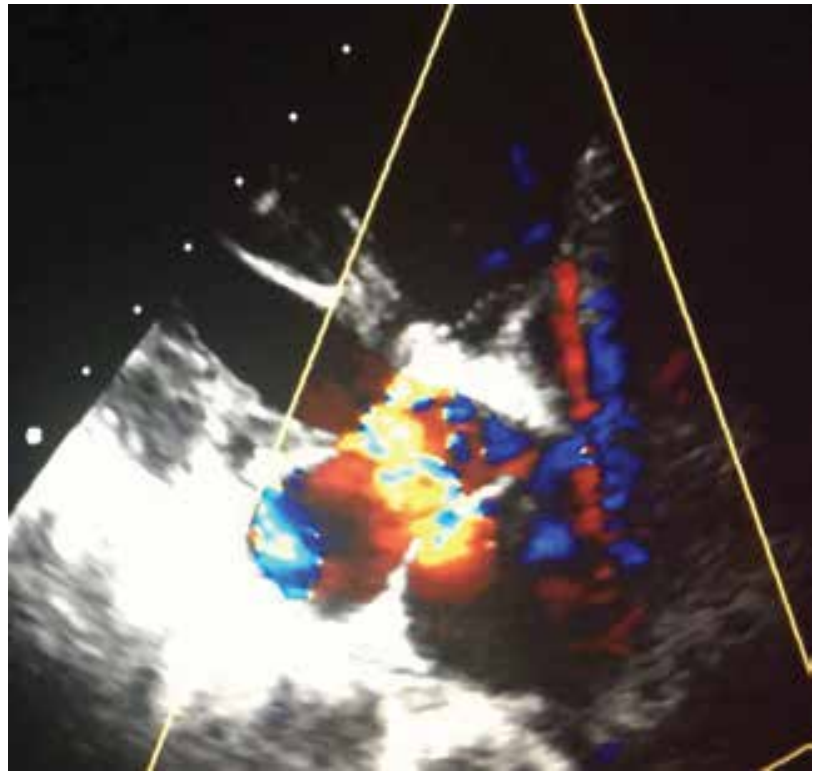

Fig 3. Colour Doppler showed flow across fenestration in cor triatriatum

\section{References}

1. Church WS. Congenital malformation of heart: abnormal septum in left auricle. Trans Path Soz. 1868;19: 188-190

2. Slight RD, Nzewi OC, Buell R, et al. Cor triatriatum sinister presenting in the adult as mitral stenosis: an analysis of factors which may be relevant in late presentation. Heart, Lung and Circulation 2005; 14(1):8-12.

3. Janet L. Eichhlz, Samer S. Hodroge. Cor-triatriatum sinister in a 43 -year-old man with syncope. Tex Heart Inst J 2013; 40(5):602-605

4. Kelmendi M, Bejiqi R, Bajraktari G, et al. Cortriatriatum sinister- three case reports. Med Arh 2009; 63(5):300-302.

5. Krasemann Z, Scheld HH, Tjan TD, et al. Cor triatriatum. Herz Kardiovaskuläre Erkrankungen 2007; 32(6):506-10.

6. Park KJ, Park IK, Sir JJ,et al. Adult cor triatriatum presenting as cardioembolic stroke. Intern Med 2009; 48 (13): 1149-52.
Cite this article as: Anish Hirachan, Dipanker Prajapati, Madhu Roka, et al. Cor Triatriatum in Adult. Nepalese Heart Journal 2017; 14(1): 33-34 